\title{
International BEAT-PCD consensus statement for infection prevention and control for primary ciliary dyskinesia in collaboration with ERN-LUNG PCD Core Network and patient representatives
}

\author{
June K. Marthin $\mathbb{B}^{1}$, Jane S. Lucas $\mathbb{B}^{2}$, Mieke Boon ${ }^{3}$, Carmen Casaulta ${ }^{4}$, Suzanne Crowley $\mathbb{1}^{5}$, \\ Damien M.S. Destouches ${ }^{6,25}$, Ernst Eber ${ }^{7}$, Amparo Escribano ${ }^{8}$, Eric Haarman ${ }^{9}$, Claire Hogg ${ }^{10}$, Bernard Maitre ${ }^{11}$, \\ Gemma Marsh $^{10}$, Vendula Martinu ${ }^{12}$, Antonio Moreno-Galdó ${ }^{13,14}$, Huda Mussaffi ${ }^{15}$, Heymut Omran ${ }^{16}$, \\ Petr Pohunek $\mathbb{1}^{12}$, Bernhard Rindlisbacher ${ }^{17,25}$, Phil Robinson $\mathbb{1}^{18,19,20}$, Deborah Snijders ${ }^{21}$, Woolf T. Walker ${ }^{2}$, \\ Panayiotis Yiallouros ${ }^{22}$, Helle Krogh Johansen $\mathbb{B}^{23,24}$ and Kim G. Nielsen ${ }^{1,24}$
}

\begin{abstract}
${ }^{1}$ Danish PCD Centre Copenhagen, Paediatric Pulmonary Service, Copenhagen University Hospital Rigshospitalet, Copenhagen, Denmark. ${ }^{2}$ Primary Ciliary Dyskinesia Centre, NIHR Respiratory Biomedical Research Centre, Clinical and Experimental Science, University of Southampton, Southampton, UK. ${ }^{3}$ Dept of Paediatrics, University Hospital Gasthuisberg, Leuven, Belgium. ${ }^{4}$ Division of Paediatric Respiratory Medicine, University Children's Hospital, Bern, Switzerland. ${ }^{5}$ Paediatric Dept of Allergy and Lung Diseases, Oslo University Hospital, Oslo, Norway. ${ }^{6}$ Association des Patients Ayant une Dyskinésie Ciliaire Primitive, Limeil-Brevannes, France. ${ }^{7}$ Division of Paediatric Pulmonology and Allergology, Dept of Paediatrics and Adolescent Medicine, Medical University of Graz, Graz, Austria. ${ }^{8}$ Pediatric Pulmonology Unit, Hospital Clínico Universitario de Valencia, University of Valencia, Valencia, Spain. ${ }^{9}$ Dept of Pediatric Pulmonology, VU University Medical Center, Amsterdam, The Netherlands. ${ }^{10}$ Depts of Paediatrics and Paediatric Respiratory Medicine, Imperial College and Royal Brompton Hospital, London, UK. ${ }^{11}$ Pulmonary Service, Centre constitutif Respirare, Centre Hospitalier intercommunal de Créteil, Univ Paris Est Creteil, INSERM, IMRB, Creteil, France. ${ }^{12}$ Paediatric Pulmonology, Paediatric Dept, 2nd Faculty of Medicine, Charles University and University Hospital Motol, Prague, Czech Republic. ${ }^{13}$ Dept of Pediatrics, Vall d'Hebron Hospital Universitari, Vall d'Hebron Barcelona Hospital Campus, Universitat Autònoma de Barcelona, Barcelona, Spain. ${ }^{14}$ CIBER of Rare Diseases (CIBERER), Instituto de Salud Carlos III (ISCIII), Madrid, Spain. ${ }^{15}$ Schneider Children's Medical Center of Israel, Petach-Tikva, Sackler School of Medicine, Tel-Aviv, Israel. ${ }^{16}$ Dept of General Paediatrics and Adolescent Medicine, University Hospital Muenster, Muenster, Germany. ${ }^{17}$ Kartagener Syndrom und Primäre Ciliäre Dyskinesie, Steffisburg, Switzerland. ${ }^{18}$ Dept of Respiratory and Sleep Medicine, Royal Children's Hospital, Parkville, Australia. ${ }^{19}$ Dept of Paediatrics, University of Melbourne, Parkville, Australia. ${ }^{20}$ Murdoch Children's Research Institute, Parkville, Australia. ${ }^{21}$ Primary Ciliary Dyskinesia Centre, Dept of Woman and Child Health (SDB), University of Padova, Padua, Italy. ${ }^{22}$ Medical School, University of Cyprus, Nicosia, Cyprus. ${ }^{23}$ Dept of Clinical Microbiology, University Hospital Rigshospitalet, Copenhagen, Denmark. ${ }^{24}$ Dept of Clinical Medicine, University of Copenhagen, Copenhagen, Denmark. ${ }^{25}$ Patient representative.
\end{abstract}

Corresponding author: June K. Marthin (june.kehlet.marthin.01@regionh.dk)

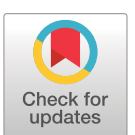

Copyright $\odot$ The authors 2021

This version is distributed under the terms of the Creative Commons Attribution NonCommercial Licence 4.0. For commercial reproduction rights and permissions contact permissions@ersnet.org
Shareable abstract (@ERSpublications)

For the first time ever, an international consensus statement for infection prevention and control in PCD is presented. A total of 20 statements were developed in a collaboration of BEAT-PCD, COST Action, ERS CRC and ERN-LUNG PCD Core Network. https://bit.ly/3yuahKt

Cite this article as: Marthin JK, Lucas JS, Boon M, et al. International BEAT-PCD consensus statement for infection prevention and control for primary ciliary dyskinesia in collaboration with ERN-LUNG PCD Core Network and patient representatives. ERJ Open Res 2021; 7: 00301-2021 [DOI: 10.1183/ 23120541.00301-2021].

\section{Abstract}

Introduction In primary ciliary dyskinesia (PCD) impaired mucociliary clearance leads to recurrent airway infections and progressive lung destruction, and concern over chronic airway infection and patient-topatient transmission is considerable. So far, there has been no defined consensus on how to control infection across centres caring for patients with PCD. Within the BEAT-PCD network, COST Action and ERS CRC together with the ERN-Lung PCD core a first initiative has now been taken towards creating such a consensus statement.

Methods A multidisciplinary international PCD expert panel was set up to create a consensus statement for infection prevention and control (IP\&C) for PCD, covering diagnostic microbiology, infection 
Received: 5 May 2021

Accepted: 16 May 2021

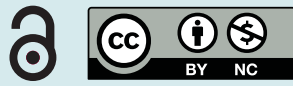

prevention for specific pathogens considered indicated for treatment and segregation aspects. Using a modified Delphi process, consensus to a statement demanded at least $80 \%$ agreement within the PCD expert panel group. Patient organisation representatives were involved throughout the process.

Results We present a consensus statement on 20 IP\&C statements for PCD including suggested actions for microbiological identification, indications for treatment of Pseudomonas aeruginosa, Burkholderia cepacia and nontuberculous mycobacteria and suggested segregation aspects aimed to minimise patient-to-patient transmission of infections whether in-hospital, in PCD clinics or wards, or out of hospital at meetings between people with PCD. The statement also includes segregation aspects adapted to the current coronavirus disease 2019 (COVID-19) pandemic.

Conclusion The first ever international consensus statement on IP\&C intended specifically for PCD is presented and is targeted at clinicians managing paediatric and adult patients with PCD, microbiologists, patient organisations and not least the patients and their families.

\section{Introduction}

Primary ciliary dyskinesia (PCD, MIM244400) is a heterogeneous recessive, or rarely dominant autosomal or X-linked disorder, exhibiting dysfunction of motile cilia causing disease in various organ systems [1]. To date, mutations in $>57$ genes are known to be associated with motile ciliopathies, the vast majority causing PCD [1, 2] and the number is continuously increasing. Dyskinetic multiple motile cilia lining the airways cause impairment of mucociliary clearance inducing recurrent and chronic infections of the upper and lower airways. Common symptoms in PCD comprise late onset respiratory distress syndrome in neonates, and chronic wet cough, recurrent and chronic otitis media with hearing impairment, sinusitis and recurrent or chronic bronchitis throughout childhood and adulthood, as well as male infertility. Persistent airway infection and inflammation lead to destructive pulmonary changes such as chronic collapsed and destroyed lobar segments and bronchiectasis [3] and may further result in end-stage lung disease, and rarely need for lung transplantation and premature death [4], thus to some extent manifestations similar to those of cystic fibrosis (CF).

Patients with PCD are frequently chronically infected with bacterial pathogens similar to those in CF [5], and in adult patients with PCD Pseudomonas aeruginosa infection has been shown to be more prevalent than Haemophilus influenzae infection [6], which underlines that $P$. aeruginosa is a pathogen of great concern also in patients with PCD and that the airway microbiology of patients with PCD seems to mirror that of CF [7]. This has enabled the extrapolation of information from CF airway microbiology to PCD, including assumptions of the need for similar management of pulmonary infections ranging from use of intensive antibiotic therapy regimens to IP\&C, despite little evidence [8-10].

Retrospective data suggest that chronic infection with $P$. aeruginosa is associated with worse lung disease in PCD, similar to what is known in CF [11]. Even though there is currently no clear evidence for patient-to-patient transmission of $P$. aeruginosa between individuals with PCD, cross-infection in PCD is still a major concern extrapolated from compelling evidence of patient-to-patient transmission in CF [12, 13], especially involving $P$. aeruginosa [14]. Failure to detect chronic infections could be caused by poor quality of airway secretion samples or due to infrequent and inconsistent sampling of airway secretion from the patients [5], or the laboratory may miss pathogens that require special culture conditions, e.g. Achromobacter species, Burkholderia species and nontuberculous mycobacteria (NTM).

Although PCD shares clinical features with CF and non-CF bronchiectasis, it is important to acknowledge the fundamental differences in pathophysiology, symptoms and prognosis between these different diseases $[14,15]$.

Healthcare providers and patient organisations sometimes encourage educational activities in PCD comprising face-to-face meetings involving multiple patients and their families, and patients could also be involved in group activities, such as pulmonary rehabilitation, where they may come into contact with other patients. However, CF patient associations/foundations have imposed strict requirements to avoid cross-infection during patient participation in different contexts [16], and we therefore recognise the importance to reflect on advice for people with PCD.

With lack of specific evidence and consensus, PCD clinicians have generally applied CF treatment principles and to some extent adult non-CF bronchiectasis principles [8]. However, a general scepticism among PCD physicians that complete adoption of CF treatment and prevention measures is appropriate has led to inconsistencies, uncertainties and generally less strict versions of strategy for patients with PCD. 
Consequently, a workshop was held at a "BEAT-PCD" academic meeting and training school in Valencia 2017 aiming to develop the framework for a consensus statement for infection prevention and control (IP\&C) for PCD to guide paediatric and adult PCD care providers, microbiologists handling specimens from patients with PCD, and patients and patient organisations, covering aspects of microbiological diagnostics, infection prevention for specific pathogens considered indicated for treatment and segregation aspects in PCD. The overall mission of "BEAT-PCD” (Better Experimental Approaches to Treat PCD; COST Action BM1407; and ERS Clinical Research Collaboration) is to promote networking and multidisciplinary clinical and translational research with the goal to improve diagnosis and treatment improving long-term outcome of patients with PCD.

The current work was also done in collaboration with the ERN-LUNG PCD core, established by the European Reference Network (https://ern-lung.eu).

The aim of these consensus statements was to provide specific suggestions as how to overall improve infection prevention and infection control in PCD, whereas specific antimicrobial therapy was considered outside the scope of this document.

Patient representatives were included throughout the process of conducting the presented consensus statements.

\section{Methods}

Developing the framework for an IP\&C consensus statement for PCD

The panel was invited with the aim of covering as many countries and experts as possible and with equal experience in paediatric and adult PCD, and at the same time with the expectation of personal attendance at the first meeting. The panel consisted of 22 experts and 2 patient representatives -20 clinicians from 16 countries including 14 paediatric pulmonologists responsible for both paediatric and adult patients, one adult chest physician exclusively managing adults with PCD, one respiratory physiotherapist, and one microbiologist; clinicians were from across Europe (Northern, Southern, Western and Eastern areas represented), the Middle East and Australia.

During the initiating face-to-face working group discussion in Valencia 2017, it was agreed that there was a need to develop a specific IP\&C for PCD and that the process should include the use of E-surveys in combination with face-to-face meetings. It was decided that the preparation and implementation of the consensus should include the following steps: 1) literature search and generation of a list of relevant items; 2) prioritising the items; and 3) discussion of these items during face-to-face meetings and voting for statements sent out via an internet-based E-survey system. The E-survey responses were to be evaluated using a modified Delphi process that differed from the original Delphi process [17] by allowing face-to-face meetings and opting out anonymity. This combined use of expert-panel face-to-face meetings and E-surveys in a modified Delphi process had previously been used successfully in the BEAT-PCD organisation for creating an international consensus statement on defining pulmonary exacerbations in PCD [18] and for developing an international consensus statement for transmission electron microscopy criteria in the diagnostics of PCD [19]. In addition, people with PCD and representatives from PCD patient organisations were to be invited to give their opinion by e-mail during the process and before finalising the consensus statement document.

The literature search was focused with an aim to support each specific statement and included search words for PCD combined with search words representing microbiological diagnostics, infection prevention and control, infection management and clinical impact of infection. The literature search was expanded to also include and combine search words for CF, non-CF bronchiectasis and for managing acute and chronic airway infections and infection in these clinical entities.

\section{The process of reaching consensus}

E-surveys

Proposed statements were presented to the PCD expert panel according to the Delphi method by use of an anonymous E-survey. For each statement, respondents could choose the answer on a Likert scale including the following terms: "strongly agree”, "agree”, "neutral”, “disagree” or "strongly disagree” or choose not to give an opinion. Respondents were encouraged to elaborate by using a comment field if they chose "neutral" or one of the disagreement possibilities to ensure future surveys could address and build on disagreements. Consensus to a statement was defined as at least $80 \%$ agreement ("strongly agree” or "agree”) among the voters within the PCD expert panel group. 
Face-to-face meetings

In between E-surveys the expert panel met for face-to-face meetings to discuss the results of agreed statements and evaluate on statements not yet agreed. After thorough discussion, each statement not yet accepted would either be modified and re-phrased or discarded.

\section{COVID-19 pandemic influence}

The consensus process ended in October 2019, prior to the start of the COVID-19 pandemic.

Owing to the worldwide restrictions and overall changed social habits caused by the COVID-19 pandemic, it was decided that statements regarding segregation issues that had not initially reached consensus should be revisited and evaluated again by the expert panel as the opinions could have changed in the light of this new situation. The statements were not re-phrased but simply re-circulated by e-mail to the PCD expert panel for re-evaluation.

\section{Results}

Over a 2-year period from September 2017 to October 2019 a group of 25 international PCD experts were invited to evaluate specific PCD consensus statements covering diagnostic microbiology, indications for infection treatment (IT) and segregation (S) aspects with the aim to create an international consensus statement for managing IP\&C in PCD.

The original IP\&C consensus work was performed before the COVID-19 pandemic and consisted of 30 proposed statements from which 19 statements reached consensus after the original consensus process that ended October 2019. Additionally, one statement regarding social behaviour and segregation in PCD settings that was initially rejected before the COVID-19 pandemic was subsequently accepted in its original form after a re-evaluation in September 2020 amidst the COVID-19 pandemic. Hence, consensus was finally reached on 20 statements that are presented here as the first ever consensus statement for IP\&C in PCD. The flow of the consensus process is illustrated in figure 1.

\section{Literature search results}

A total of 11 studies were retrieved from the literature search that concerned prevalence, clinical impact and management of $P$. aeruginosa infection in patients with PCD [5, 6, 9, 11, 20-26].

Prevalence of $P$. aeruginosa in patients with PCD ranged from $9 \%$ to $47 \%$, the prevalence increasing with age [5, 23-25].

Three observational studies showed worse computed tomography (CT) scan scores in patients with PCD chronically infected with $P$. aeruginosa compared to non-infected patients $[6,11,20]$, whereas convincing impact of $P$. aeruginosa infection on lung function was not consistently found among four included studies [11, 23, 25], as only one study out of four showed decreased forced expiratory volume in $1 \mathrm{~s}$ $\left(\mathrm{FEV}_{1}\right)$ in $P$. aeruginosa-infected patients.

One study based on an online survey questionnaire described considerable variation in how P. aeruginosa infection was managed across 43 PCD centres in 26 European countries and also that only a few centres had written guidelines for PCD management [9].

Two studies were concerning prevalence of NTM infection in PCD and reported a prevalence of 3\% in children [23] and $10 \%$ in adults [24], respectively.

Achromobacter xylosoxidans was shown to be a pathogen primarily identified among adult patients with PCD in a single study of a mixed population including both children and adults with PCD in which A. xylosoxidans was recovered in $6 \%$ of samples from adults older than 25 years versus only in $1 \%$ of samples from children below 12 years of age [5]. No other studies were identified in the literature search that concerned prevalence, impact or management of A. xylosoxidans.

No studies were identified in the literature search that concerned prevalence, impact or management of Burkholderia cepacia in PCD.

There were no studies identified concerning IP\&C and control of lung infections in PCD. 
4 proposed statements were excluded early in the consensus process

Working Group formation by invitation in Valencia 2017

Appointing PCD expert panel and setting up an IP\&C for PCD

\section{$\checkmark$}

Face-to-face meeting in Milan 2017

Discussion on proposed statements before sending out first E-survey

Discussion on proposed statements bef

\section{E-Survey 1}

30 proposed statements sent out to PCD expert panel members

11 agreed statements

Face-to-face meeting in Lisbon 2018

Discussion of E-Survey 1 results and re-phrasing for E-Survey 2

E-Survey $2^{\#}$
15 re-phrased proposed statements sent out to PCD expert panel members

\section{4 agreed statements}

15 re-phrased proposed statements sent out to PCD expert panel members

Face-to-face meeting in Poznan 2019

Discussion of E-Survey 2 results and re-phrasing for E-Survey 3

E-Survey $3^{\#}$

10 re-phrased proposed statements sent out to PCD expert panel members

$\longrightarrow 4$ agreed statements

A total of 7 proposed

statements were finally

rejected after E-survey

$1+2+3($ table 2$)$

\begin{tabular}{l|l|l} 
Face-to-face meeting in Madrid 2019 & \\
Working Group presents 19 final specific IP\&C PCD statements & agreed statement (wearing of face
\end{tabular}

mask and keeping social distance)

$$
1
$$

$\checkmark$

September 2020 after hit of COVID-19 pandemic

Re-evaluation of previous rejected statements regarding segregation

PCD expert panel agreed on 20

final specific IP\&C PCD statements

in September 2020 (table 1)

FIGURE 1 Consensus process of the infection prevention and control (IP\&C) for primary ciliary dyskinesia (PCD) international consensus statement using a modified Delphi process including both E-surveys and face-to-face meetings. A total of 20 statements were agreed for the final IP\&C statement (presented in table 1). A total of seven proposed statements were excluded after finalising the process of E-survey $1+2+3$ and the face-to-face meetings (presented in table 2). A total of four proposed statements were excluded early on from the further consensus process. \#: during the process of re-phrasing some statements were either split up or combined. 
TABLE 1 Final 20 suggested BEAT-PCD consensus statements included in the infection prevention and control for primary ciliary dyskinesia (PCD) statements

No. Aspect Statement

Consensus E-survey $\% \quad$ voters

\begin{tabular}{|c|c|c|c|c|}
\hline 1 & DM & $\begin{array}{l}\text { "The BEAT-PCD network suggests to culture on selective media for Pseudomonas aeruginosa routinely in } \\
\text { every airway secretion sample" }\end{array}$ & 100 & 18 \\
\hline 2 & DM & "The BEAT-PCD network suggests that all PCD Centres have access to bacterial typing" & 100 & 14 \\
\hline 3 & DM & $\begin{array}{l}\text { "The BEAT-PCD network suggests to culture airway secretion samples" from patients at least } 4 \text { times } \\
\text { annually" }\end{array}$ & 94.4 & 18 \\
\hline 4 & DM & $\begin{array}{l}\text { "The BEAT-PCD network suggests to culture for NTM at least annually and in addition at any unexplained } \\
\text { deterioration of lung function" }\end{array}$ & 92.9 & 14 \\
\hline 5 & DM & "The BEAT-PCD network suggests routine bacterial typing at first positive culture of Burkholderia cepacia" & 92.9 & 14 \\
\hline 6 & DM & $\begin{array}{l}\text { "The BEAT-PCD network suggests the use of modified Leeds criteria [5] when defining chronicity of } \\
\text { Pseudomonas aeruginosa" }\end{array}$ & 83.3 & 18 \\
\hline 7 & IT & $\begin{array}{l}\text { "The BEAT-PCD network suggests that treatment of NTM relies on 1) Pulmonary symptoms and 2) Nodular } \\
\text { or cavitary processes on chest radiograph and/or bronchiectasis with small nodules on HRCT scan and } \\
\text { 3) Positive culture results from at least two separate airway secretion samples" or positive culture results } \\
\text { from at least one bronchial wash or lavage or mycobacterial histological findings in either transbronchial } \\
\text { or lung biopsy material together with positive microbiological culture according to } 2020 \text { ATS/IDSA } \\
\text { criteria” [27] }\end{array}$ & 100 & 18 \\
\hline 8 & IT & $\begin{array}{l}\text { "The BEAT-PCD network suggests that cultured Pseudomonas aeruginosa is treated regardless of symptoms } \\
\text { and microscopy" }\end{array}$ & 100 & 18 \\
\hline 9 & IT & $\begin{array}{l}\text { "The BEAT-PCD network suggests that Haemophilus influenzae, Moraxella catarrhalis, Streptococcus } \\
\text { pneumoniae, Staphylococcus aureus (MSSA) are treated, if the patient is symptomatic" }\end{array}$ & 94.4 & 18 \\
\hline 10 & IT & $\begin{array}{l}\text { "The BEAT-PCD network suggests that cultured Multi-Resistant Staphylococcus aureus (MRSA) is treated } \\
\text { regardless of symptoms and microscopy" }\end{array}$ & 86 & 14 \\
\hline 11 & IT & $\begin{array}{l}\text { "The BEAT-PCD network suggests that cultured Burkholderia cepacia complex is treated regardless of } \\
\text { symptoms and microscopy" }\end{array}$ & 83.3 & 18 \\
\hline 12 & $S$ & $\begin{array}{l}\text { "The BEAT-PCD network suggests that patients with Pseudomonas aeruginosa infection should be } \\
\text { segregated in outpatient and in-patient settings" }\end{array}$ & 100 & 18 \\
\hline 13 & $S$ & $\begin{array}{l}\text { "The BEAT-PCD network suggests that patients with NTM infection should be segregated in outpatient and } \\
\text { in-patient settings" }\end{array}$ & 94.4 & 18 \\
\hline 14 & $S$ & $\begin{array}{l}\text { "The BEAT-PCD network suggests that patients with MRSA infection should be segregated in outpatient } \\
\text { and in-patient settings" }\end{array}$ & 88.9 & 18 \\
\hline 15 & $S$ & $\begin{array}{l}\text { "The BEAT-PCD network suggests that patients with Burkholderia cepacia complex infection should be } \\
\text { segregated in outpatient and in-patient settings" }\end{array}$ & 88.9 & 18 \\
\hline 16 & $S$ & $\begin{array}{l}\text { "The BEAT-PCD network suggests that all PCD centres have written guidelines for segregation that are } \\
\text { adapted to the facilities of the individual centres and to the best standards" }\end{array}$ & 94.4 & 18 \\
\hline 17 & $\mathrm{~S}^{+}$ & $\begin{array}{l}\text { "During the COVID-19 Pandemic concerning arrangements outside the hospital: The BEAT-PCD network } \\
\text { suggests (e.g., BEAT-PCD Conferences) that if more than one patient with PCD is attending indoor events } \\
\text { they should keep at least a 2-m distance and wear a mask regardless of infection status" }\end{array}$ & 93.8 & $\begin{array}{l}16 \\
\text { e-mail } \\
\text { respondents }\end{array}$ \\
\hline 18 & $\mathrm{~S}$ & $\begin{array}{l}\text { "The BEAT-PCD network suggests that patients with identified viral infection or 'clinically having a cold" } \\
\text { should be temporarily segregated in outpatient and in-patient settings, or at least wear a mask" }\end{array}$ & 88.2 & 17 \\
\hline 19 & $S$ & $\begin{array}{l}\text { "The BEAT-PCD network suggests a cleaning procedure between patients and at the end of the day to } \\
\text { include registered hospital-grade disinfectant/detergent" }\end{array}$ & 83.3 & 18 \\
\hline 20 & $S$ & $\begin{array}{l}\text { "The BEAT-PCD network suggests that the following bacteria do not need specific considerations regarding } \\
\text { segregation: Haemophilus influenzae, Moraxella catarrhalis, Streptococcus pneumoniae and } \\
\text { Staphylococcus aureus (MSSA)" }\end{array}$ & 82.4 & 17 \\
\hline
\end{tabular}

DM: diagnostic microbiology aspects; NTM: nontuberculous mycobacteria; IT: infection treatment aspects; HRCT: high-resolution computed tomography; S: segregational aspects; COVID-19: coronavirus disease 2019; MSSA: methicillin-susceptible Staphylococcus aureus. Voters: 18 PCD experts responded to the E-surveys; 16 voted by e-mail for consensus statement \#15. \#: Airway secretion sample defined as: sputum sample or oropharyngeal cough swab or laryngeal suction. ": "Symptomatic" = increased symptoms judged at the discretion of the treating physician. ${ }^{+}$: Accepted consensus after re-evaluation due to COVID-19 pandemic. Accepted consensus was based on the decisions from an international PCD expert panel. Each proposed statement was presented for the expert panel using a Likert scale offering five possible response options: "Strongly agree", "Agree", "Neutral", "Disagree", "Strongly disagree". Consensus for a proposed statement was defined where at least $80 \%$ agreement ("Strongly agree" or "Agree") was obtained within the PCD expert panel.

Initiating face-to-face meeting: BEAT-PCD Valencia meeting April 2017

At this initiating meeting 17 PCD experts participated. Another eight experts who were absent were subsequently invited to join the process by e-mail. The 17 experts attending the meeting were asked whether they agreed on the need for developing a consensus for IP\&C for PCD and 14 out of 17 (82\%) 


\begin{tabular}{|c|c|c|c|c|}
\hline No. & Aspect & Statement & $\begin{array}{l}\text { Consensus } \\
\%\end{array}$ & Voters \\
\hline 1 & $\mathrm{DM}$ & $\begin{array}{l}\text { "The BEAT-PCD network suggests use of Gram-staining and microscopy as part of clinical microbiological } \\
\text { diagnostics at PCD centres where this service is available" }\end{array}$ & 78.6 & $14 / 18$ \\
\hline 2 & DM & $\begin{array}{l}\text { "The BEAT-PCD network suggests investigating for viral respiratory infections in case of a clinical } \\
\text { exacerbation" }\end{array}$ & 72.8 & $18 / 18$ \\
\hline 3 & DM & $\begin{array}{l}\text { "The BEAT-PCD network suggests routine bacterial typing at first positive culture of Achromobacter } \\
\text { xylosoxidans" }\end{array}$ & 71.4 & $14 / 18$ \\
\hline 4 & DM & $\begin{array}{l}\text { "The BEAT-PCD network suggests routine bacterial typing at first positive culture of Pseudomonas } \\
\text { aeruginosa" }\end{array}$ & 57.2 & $14 / 18$ \\
\hline 5 & IT & $\begin{array}{l}\text { "The BEAT-PCD network suggests that cultured Achromobacter xylosoxidans is treated regardless of } \\
\text { symptoms and microscopy" }\end{array}$ & 66.7 & $18 / 18$ \\
\hline 6 & $S$ & $\begin{array}{l}\text { "The BEAT-PCD network suggests that patients with Achromobacter xylosoxidans should be segregated in } \\
\text { outpatient and in-patient settings" }\end{array}$ & 77.8 & $18 / 18$ \\
\hline 7 & $S$ & $\begin{array}{l}\text { "The BEAT-PCD network suggests to always have a 30-minute wait between all PCD patients regardless of } \\
\text { infection status" }\end{array}$ & 72.8 & $18 / 18$ \\
\hline
\end{tabular}

Seven proposed consensus statements did not reach consensus during the Delphi process. Each proposed statement was presented for the expert panel using a Likert scale offering five possible response options: "Strongly agree", "Agree”, "Neutral”, "Disagree”, "Strongly disagree”. "No consensus" was defined as $<80 \%$ agreement ("Strongly agree" or "Agree") within the PCD expert panel. DM: diagnostic microbiology aspects; IT: infection treatment aspects; S: segregational aspects. Voters: 18 PCD experts responded to the E-surveys.

agreed on this. Next, the participants were asked whether to adopt all suggestions from the already existing IP\&C for CF [16] and include those for PCD as well. Only 59\% agreed on this, i.e., less than the goal of achieving $80 \%$ agreement. Based on this, it was decided to undertake a survey aiming to develop a separate IP\&C for PCD.

\section{E-surveys and face-to-face meetings}

At a BEAT-PCD meeting in Milan, September, 2017 during the ERS International Congress the expert panel had a face-to-face meeting where initial proposed statements were discussed before sending out the first E-survey.

E-survey 1 resulted in agreement in 11 of the proposed statements.

Following E-survey 1 a face-to-face meeting was held in Lisbon, February, 2018. The results from the first E-survey were presented for the expert panel and altered phrasing of statements not yet agreed upon was discussed for the following E-survey 2.

Four statements were further excluded after evaluating E-survey 1; reasons for exclusion are given below.

E-survey 2 resulted in acceptance of four additional statements, thereby increasing consensus to a total of 15 presented statements.

Face-to-face meeting Poznan, March, 2019: Each of the remaining statements that had not reached agreement after E-survey 2 were presented to the expert panel again. Based on comments from the expert panel 10 re-phrased statements were circulated for a third and final E-survey.

E-survey 3: Four additional statements reached agreement within the expert panel, increasing the consensus to 19 statements.

Face-to face meeting Madrid, October, 2019 at a BEAT-PCD meeting held during the ERS International Congress: The 19 final statements were agreed for the IP\&C consensus statement.

COVID-19 influence: E-mail correspondence with the PCD expert panel during the COVID-19 pandemic, September, 2020. One previously rejected statement regarding segregation now reached consensus after e-mail re-visiting of statements regarding segregation. This lastly accepted statement involved suggested wearing of a face mask and keeping a distance of at least $2 \mathrm{~m}$ during indoor patient meetings. 
With that a total of 20 consensus statements were included for the overall final IP\&C for PCD (figure 1).

\section{Accepted consensus statements}

Altogether, consensus was reached for 20 out of originally 30 proposed statements (66.7\%). Complete agreement, defined as $100 \%$ consensus among voters was achieved on 5 out of 20 agreed statements (25\%), and general consensus defined as at least $80 \%$ agreement was achieved on 15 out of 20 (75\%) of agreed statements. Detailed results on agreed consensus statements are summarised in table 1.

In total, consensus was reached on six items considering diagnostic microbiology aspects, five items considering indication for infection treatment (IT) aspects and nine items considering segregation (S) aspects. Consensus on the segregation aspect items increased from eight to nine after three selected originally proposed and discarded statements were revisited after the start of the COVID-19 pandemic.

\section{BEAT-PCD suggestions concerning diagnostic microbiology (DM) aspects}

Consensus was reached concerning a number of measures taken to limit infections from opportunistic pathogens such as $P$. aeruginosa, B. cepacia and NTM, including early detection of infection by suggesting at least four airway secretion samples obtained from PCD patients annually as per modified Leeds criteria for defining chronic $P$. aeruginosa infection [5], and suggesting specific microbiological laboratory measures such as extended culture on selective media and access to bacterial typing in order to increase microbiological diagnostic accuracy.

\section{BEAT-PCD suggestions concerning indication for infection treatment (IT) aspects}

Consensus was reached towards suggestion of treating $P$. aeruginosa, B. cepacia and methicillin-resistant Staphylococcus aureus (MRSA) regardless of symptoms and NTM according to ATS/ERS/ESCMID/IDSA criteria from the 2020 guideline [27], and treatment of airway secretion positive patients with $H$. influenzae, Moraxella catarrhalis, Streptococcus pneumoniae and S. aureus (MSSA) only if symptomatic. Consensus for treatment of MRSA followed common guidelines for MRSA eradication [28].

\section{BEAT-PCD suggestions concerning segregation (S)}

Consensus was reached towards need for segregation if patients were infected with $P$. aeruginosa and B. cepacia complex, MRSA and NTM, whereas no consensus was obtained for suggesting segregation in patients infected with $A$. xylosoxidans or patients with positive culture results for $H$. influenzae, M. catarrhalis, S. pneumoniae and S. aureus (MSSA).

Social distancing of at least $2 \mathrm{~m}$ between patients with PCD and patient use of face masks during indoor meetings including more than one patient with PCD became the suggestion after the outbreak of COVID-19, although this was initially rejected during the pre-COVID-19 consensus process.

\section{Rejected statements}

Eventually, seven proposed statements remained unagreed and were excluded from the final consensus statement (table 2). Of particular note we achieved no consensus for indication of treatment or segregation in case of positive culture of A. xylosoxidans.

\section{Excluded statements}

Four proposed statements were excluded early in the process after E-survey 1.

E-survey 1 included a statement suggesting implementing the existing IP\&C for CF [16] for PCD. However, this was turned down as only 9 out of 17 voters agreed (52.9\%), i.e. less than the goal of $80 \%$ agreement and thus supporting the development of a specific IP\&C for PCD. Therefore, this particular statement was excluded from the further consensus process. Two proposed statements regarding indication for treatment and segregation if infected with Stenotrophomonas maltophilia were both turned down in E-survey 1 (29.4\% agreement and 23.5\% agreement, respectively) and excluded by agreement among the expert panel members at the face-to-face meeting following the E-survey 1. The primary reason for the exclusion was that S. maltophilia is considered a possible "bystander" with little known pathogenic potential even in patients with CF [29-31]. The fourth excluded statement that proposed routine bacterial typing of $S$. aureus if a patient had three or more positive cultures per year was turned down in E-survey 1 (50\% agreement) and thus excluded, as it was considered mainly of research interest among the PCD expert panel members. 
Discussion

Since the description of PCD as a unique disease entity in 1976 [32, 33], management strategies for PCD have been derived mainly from CF care. This has been considered acceptable, as both represent conditions with impaired mucociliary airway clearance, and since evidence-based studies designed specifically for treating PCD have been completely lacking until very recently [34, 35]. Now, with the growing knowledge of the pathophysiology and natural history of PCD, and the fact that PCD is definitively different from that of CF, the time has come to create a separate IP\&C consensus statement in PCD.

In this consensus statement, we present 20 suggestions regarding microbiology diagnostics, indications for infection treatment and segregation actions, aiming specifically for better IP\&C in patients with PCD including separate suggestions for infection prevention for specific pathogens considered indicated for treatment: P. aeruginosa, B. cepacia and NTM as well as $H$. influenzae, S. pneumoniae, $M$. catharrhalis and MSSA/MRSA. Our statement also provides general suggestions for cleaning procedures and segregation aspects in PCD care.

\section{BEAT-PCD suggestions concerning monitoring and indications for treatment of bacterial airway infections}

A general concern for chronic $P$. aeruginosa infection in PCD is rational. A substantial prevalence of $P$. aeruginosa has been reported in PCD cohorts [5, 23-25], and there are indications of worse CT scan scores in chronically infected patients with PCD $[11,20]$ and a risk of further decline of lung function when chronically infected with $P$. aeruginosa [11]. Still, the evidence from studies including patients with PCD and $P$. aeruginosa infection are sparse and hence our presented suggestions for treating $P$. aeruginosa infections were additionally based on the indications of CF and PCD parallelism in airway susceptibility and bacterial adaptation, as the underlying genetic defects in these two diseases result in impaired mucociliary clearance [36]. Both diseases lead to increased susceptibility to airway infections, e.g. H. influenzae, S. aureus and P. aeruginosa. The infections are characterised by adaptation of the bacterial populations to the site of infection.

In two studies using whole genome sequencing of longitudinally collected $P$. aeruginosa isolates from patients with CF and PCD, evidence was found for phenotypic and genotypic parallelism in the evolution of $P$. aeruginosa across infected patients. The parallel changes and convergent adaptation and evolution are believed to be caused by similar selective forces such as the intensive antibiotic treatment and the inflammatory response, which drive the evolutionary processes [12, 22]. In a selected cohort of $34 \mathrm{CF}$ patients and 12 patients with PCD similar convergent evolution was identified in eight genes related to antibiotic resistance, quorum sensing, motility, Type III secretion and mucoidity [12, 22].

All in all, the concern of clinical impact together with the predicted parallelism in bacterial behaviour in CF and PCD patients justified a well-founded concern of $P$. aeruginosa infection and is now reflected in the present BEAT-PCD consensus statement for close airway secretion monitoring, indication for treatment and segregation of patients infected with $P$. aeruginosa to limit its spread. Parallel suggestions for treatment of $B$. cepacia were proposed, primarily due to a concern derived from $\mathrm{CF}$ experience, as B. cepacia has only been rarely described in PCD and no studies on PCD and B. cepacia infection were identified in our literature search.

For the treatment of NTM, BEAT-PCD agreed to adopt the criteria from the ATS/ERS/ESCMID/IDSA guideline [27] as described in table 1. In alignment with these treatment criteria, BEAT-PCD also suggests culturing for NTM at least annually [37] and at the time of any unexplained deterioration of lung function.

Consensus was not reached on suggesting indication for treatment or segregation of patients with a positive culture for A. xylosoxidans, the main argument for rejection being the rarity of documented A. xylosoxidans infection in PCD, no published studies on A. xylosoxidans infection in PCD and overall lack of experience within the expert panel group. In CF, chronic infection with $A$. xylosoxidans has been associated with lung function decline [38, 39] and patient-to-patient transmission [40, 41], which raises the concern that A. xylosoxidans infection may also play an ignored but significant role in PCD. Future studies to investigate the impact of $A$. xylosoxidans in PCD are certainly warranted.

BEAT-PCD suggestions concerning adoption of Leeds criteria for defining chronicity of $\mathrm{P}$. aeruginosa The most commonly used definition for $P$. aeruginosa status in CF epidemiological research is that from the Leeds criteria [7], and although it may not be optimal in all circumstances and may, in some cases, lead to the misinterpretation of a chronic infection for an intermittent infection [42, 43], these criteria were considered as the most suitable definition at present. 
The expert panel decided to adopt a modified version [5] of the original Leeds criteria established for CF [7] to define chronicity of P. aeruginosa in the current IP\&C statement for PCD. The original Leeds criteria [7] define "chronic infection" when $>50 \%$ of the preceding 12 months' cultures are positive for the specific pathogen and "intermittent infection" when $50 \%$ or less of the preceding 12 months' cultures have been positive for the specific pathogen and "free of infection" when no growth has occurred in the lungs in the previous 12 months. According to the modification of these criteria used in the current statements, the patient should provide at least four samples of airway secretion per year [5].

Within the expert panel concerns were raised for not being able to diagnose chronic infections due to poor quality of airway secretion samples or due to infrequent and inconsistent sampling of airway secretion. An additional concern was that the sinuses in patients with PCD can function as a bacterial reservoir for recurrent lung infection, including hosting $P$. aeruginosa [21]. The suggested requirement of four annual samples of airway secretion is in alignment with previous recommendations for pulmonary care and monitoring for PCD [10]. Although it may seem difficult to achieve, the expert panel believes that regular monitoring is the only way to detect, for example, $P$. aeruginos $a$ at the earliest timepoint possible and have chosen to keep the bar at this higher level in recognition that an even more frequent monitoring is certainly further optimal, but probably difficult to implement.

\section{BEAT-PCD suggestions concerning segregation inside and outside hospital settings}

The BEAT-PCD IP\&C consensus statement suggests segregation inside and outside hospital settings if the patient has a known infection with $P$. aeruginosa, B. cepacia, NTM or MRSA even at first positive isolate.

At present there is no clear evidence for patient-to-patient transmission of $P$. aeruginosa between patients with PCD. However, transmission of non-susceptible strains of $P$. aeruginosa between CF patients has been a well-known problem since first described in 1986 [44]. From the experience in CF, it is also known that some clone types have the potential to spread to other patients, e.g. patients with COPD. Therefore, all direct contact between individuals with CF is generally suggested to be avoided whether inside or out of the hospital [45, 46].

Since at least four samples of airway secretion per year is also suggested, the last positive airway secretion culture could be 3 months old or more. Hence, there will be a potential risk for transmission of these unwanted pathogens if they were contracted more recently than the last airway secretion sample. A consideration to minimise this risk could be to ask patients to send their airway secretion sample a week before their outpatient appointment to allow any change of infection status to be known on the day of the patients' appointments. It should, however, be noted that slow-growing pathogens in a sample may bias culture results owing to overgrowth of more fast-growing bacteria with the risk of not detecting the pathogenic bacteria if present in small amounts.

A 30-min gap between all PCD patient appointments regardless of infectious status that could otherwise minimise transmission did not reach consensus as it was perceived too difficult to adhere to due to logistics at various sites.

It has been suggested that non-CF bronchiectasis patients managed within a CF service centre should be managed according to the same strict infection control procedures as patients with CF, because of the risk of transmission from the CF patients to the non-CF bronchiectasis patients and vice versa [14]. This concern for transmission was not evaluated in the current BEAT-PCD IP\&C statement. However, shared facilities between patients with CF and those with PCD do occur in some of the PCD centres, and adherence to the strict CF segregation rules should be considered in these centres.

The statements on segregation are more far-reaching based on a precautionary principle and more detailed with regard to both bacteria and viruses and in line with IP\&C for CF [16] and clearly deviate from recommendations regarding bronchiectasis in general [47].

\section{BEAT-PCD suggestions concerning cleaning procedures in hospital settings}

Cleaning of the patient-room between appointments and at the end of the day with registered hospital-grade disinfectant/detergent was suggested to avoid the general spread of microorganisms to other patients. According to World Health Organization (WHO) guidelines, healthcare personnel must also perform hand hygiene before entering the room and when leaving the room of a patient, before or after direct contact with a patient, before putting gloves on and after removing gloves, for sterile and nonsterile procedures, after contact with a patient's skin, mucous membranes, respiratory secretions or other body fluids, and after contact with medical equipment near the patient that may be contaminated with respiratory secretions [48]. 
BEAT-PCD suggestions concerning wearing a mask and keeping a 2-m distance at outside hospital patient events

The overall concern for cross-infection of, for example, $P$. aeruginosa between patients with PCD led to this proposed statement. Use of face masks had previously been shown to be effective at reducing the release of potentially infective Pseudomonas-containing aerosols in patients with CF [49,50], and wearing a face mask is suggested for CF in healthcare settings [51]. However, our PCD patient representatives raised concerns of stigmatisation by wearing masks and also that highly valued meetings between patients outside the hospital would be too difficult to organise, and therefore the opportunity for mutual support and face-to-face exchange of experiences could get lost. Partly due to this patient concern, the proposed statement was initially rejected but later accepted following the COVID-19 outbreak. Currently, during the COVID-19 pandemic, wearing a mask has become commonly suggested and widely used among most people in all daily life situations and hence stigmatism is currently not an issue. In addition, many larger patient meetings will currently not be possible due to the general assembly restrictions in most countries. It is, however, important to stress that this issue had been clearly controversial before the COVID-19 pandemic, since both PCD experts and patient's representatives were reluctant to accept the suggestion. Whether the suggestion should be removed from the statement once the COVID-19 pandemic is under full control remains open, and the board of PCD experts and patient's representatives should be consulted again when this time comes [52].

\section{Limitations of the study}

Members of the expert panel were mainly paediatric pulmonologists, whereas representation from physicians managing adult PCD was low, which could lead to the concern that adult patients were not appropriately represented. However, adult PCD care has traditionally been managed by paediatricians in many PCD centres for many years, and almost $75 \%$ of the medical experts in the present consensus panel have extensive experience in management of both children and adults with PCD.

Many of the consensus statements grew out of extrapolation of evidence based on CF because of the lack of current evidence in the field of PCD, although extrapolation from a whole different disease entity such as CF can never be optimal. Crafting evidence specifically for PCD is of extreme importance and will also be possible in the near future owing to currently growing PCD registries, more randomised controlled trials to come and further multicenter observational studies in PCD cohorts. In the current situation and for lack of anything better, we found that the Delphi method used was appropriate in achieving the presented statements concerning PCD.

There is currently no evidence regarding patient-to-patient transmission of NTMs and no evidence regarding the risk associated with infection of specific NTM species in PCD. However, from the experience across the expert panel it was clear that NTMs indeed are sometimes cultured and also in some cases cause clinical symptoms and radiological changes in patients with PCD. Based on these experiences, the expert panel chose to include statements suggesting indication of treatment as per ATS/ERS/ESCMID/ IDSA 2020 guidelines [27], which include treatment strategies for a number of NTM species. In alignment with this and due to lack of evidence in PCD we chose not to differentiate between specific NTMs in the statements. Similarly, the expert panel chose to suggest segregation whenever any NTM species is cultured.

We consider these IP\&C statements as a guide for all physicians who manage paediatric and adult patients with PCD, whether it takes place in a dedicated PCD centre, a centre where the experts in addition to CF also manage PCD or where PCD patients constitute a minority among larger numbers of patients with all sorts of other diagnoses within adult pulmonary medicine.

\section{Conclusion}

We here provide the first International BEAT-PCD IP\&C consensus statement for PCD within COST Action and ERS Clinical Research Collaboration together with the ERN-LUNG PCD Core Network.

The consensus statement consists of 20 suggestions for IP\&C intended specifically for patients with PCD in a number of settings. The statements are targetted at paediatric and adult PCD healthcare providers, microbiologists, PCD patient organisations and patients themselves. Patient representatives were included throughout the process of conducting the presented consensus statements. The statements include suggestions for microbial diagnostics, infection prevention for specific pathogens considered indicated for treatment, and segregation strategies for outpatient and inpatient settings as well as for non-healthcare settings. 
Acknowledgements: We wish to thank the PCD patient organisations that closely collaborated with us. UK: PCD Family Support Group UK. France: Association des Patients Ayant une Dyskinésie Ciliaire Primitive, France. Germany and Switzerland: Kartagener Syndrom und Primäre Ciliäre Dyskinesie (www.kartagener-syndrom.org). Denmark: The PCD Network affiliated with The Lung Association, Denmark.

Conflict of interest: J.K Marthin has nothing to disclose. J.S. Lucas has nothing to disclose. M. Boon has nothing to disclose. C. Casaulta has nothing to disclose. S. Crowley has nothing to disclose. D.M.S. Destouches has nothing to disclose. E. Eber has nothing to disclose. A. Escribano has nothing to disclose. E. Haarman has nothing to disclose. C. Hogg has nothing to disclose. B. Maitre has nothing to disclose. G. Marsh has nothing to disclose. V. Martinu has nothing to disclose. A. Moreno-Galdó reports a personal payment for a lecture from Abbvie, travel support from Abbvie and Novartis, and personal payments for advisory boards from Abbvie and Sanofi. H. Mussaffi has nothing to disclose. H. Omran has nothing to disclose. P. Pohunek has nothing to disclose. B. Rindlisbacher has nothing to disclose. P. Robinson has nothing to disclose. D. Snijders has nothing to disclose. W.T. Walker has nothing to disclose. P. Yiallouros has nothing to disclose. H.K. Johansen has nothing to disclose. K.G. Nielsen has nothing to disclose.

Support statement: The BEAT-PCD network was initially funded by COST Action BM 1407, and now by European Respiratory Society Clinical Research Collaboration. Most participating researchers and contributors are participants in European Reference Network for Rare Respiratory Diseases (ERN-LUNG) project identification number 739546. Petr Pohunek and Vendula Martinu were supported by Ministry of Health of the Czech Republic grant number NV-19-07-00210. All rights reserved. First and last author were supported by The Lung Association, Denmark and Børnelungefonden. Funding information for this article has been deposited with the Crossref Funder Registry.

\section{References}

$1 \quad$ Wallmeier J, Nielsen KG, Kuehni CE, et al. Motile Ciliopathies. Nat Rev Dis Primers 2020; 6: 77.

2 Lucas JS, Davis SD, Omran H, et al. Primary ciliary dyskinesia in the genomics age. Lancet Respir Med 2020; 8: 202-216.

3 Davis SD, Rosenfeld M, Lee HS, et al. Primary ciliary dyskinesia: longitudinal study of lung disease by ultrastructure defect and genotype. Am J Respir Crit Care Med 2019; 199: 190-198.

4 Hayes D, Reynolds SD, Tumin D. Outcomes of lung transplantation for primary ciliary dyskinesia and Kartagener syndrome. J Heart Lung Transplant 2016; 35: 1377-1378.

5 Alanin MC, Nielsen KG, von Buchwald C, et al. A longitudinal study of lung bacterial pathogens in patients with primary ciliary dyskinesia. Clin Microbiol Infect 2015; 21: 1093.e1-1093.e7.

6 Shah A, Shoemark A, MacNeill SJ, et al. A longitudinal study characterising a large adult primary ciliary dyskinesia population. Eur Respir J 2016; 48: 441-450.

7 Lee TW, Brownlee KG, Conway SP, et al. Evaluation of a new definition for chronic Pseudomonas aeruginosa infection in cystic fibrosis patients. J Cyst Fibros 2003; 2: 29-34.

8 Barbato A, Frischer T, Kuehni CE, et al. Primary ciliary dyskinesia: a consensus statement on diagnostic and treatment approaches in children. Eur Respir J 2009; 34: 1264-1276.

9 Crowley S, Holgersen MG, Nielsen KG. Variation in treatment strategies for the eradication of Pseudomonas aeruginosa in primary ciliary dyskinesia across European centers. Chron Respir Dis 2019; 16: 1479972318787919.

10 Shapiro AJ, Zariwala MA, Ferkol T, et al. Diagnosis, monitoring, and treatment of primary ciliary dyskinesia: PCD Foundation Consensus Recommendations based on state of the art review. Pediatr Pulmonol 2016; 51: 115-132.

11 Cohen-Cymberknoh M, Weigert N, Gileles-Hillel A, et al. Clinical impact of Pseudomonas aeruginosa colonization in patients with primary ciliary dyskinesia. Respir Med 2017; 131: 241-246.

12 Marvig RL, Sommer LM, Molin S, et al. Convergent evolution and adaptation of Pseudomonas aeruginosa within patients with cystic fibrosis. Nat Genet 2015; 47: 57-64.

13 Parkins MD, Somayaji R, Waters VJ. Epidemiology, biology, and impact of clonal Pseudomonas aeruginosa infections in cystic fibrosis. Clin Microbiol Rev 2018; 31: e00019-18.

14 Chalmers JD, Ringshausen FC, Harris B, et al. Cross-infection risk in patients with bronchiectasis: a position statement from the European Bronchiectasis Network (Embarc), EMBARC/ELF Patient Advisory Group and European Reference Network (ERN-Lung) Bronchiectasis Network. Eur Respir J 2018; 51: 1701937.

15 Robinson P, Morgan L. Bronchiectasis in PCD looks different to CF on CT scan. Multidiscip Respir Med 2018; 13: 24 .

16 Saiman L, Siegel JD, LiPuma JJ, et al. Infection prevention and control guideline for cystic fibrosis: 2013 update. Infect Control Hosp Epidemiol 2014; 35 Suppl 1: S1-S67.

17 Murphy MK, Black NA, Lamping DL, et al. Consensus development methods, and their use in clinical guideline development. Health Technol Assess 1998; 2: i-iv, 1. 
18 Lucas JS, Gahleitner F, Amorim A, et al. Pulmonary exacerbations in patients with primary ciliary dyskinesia: an expert consensus definition for use in clinical trials. ERJ Open Res 2019; 5: 00147-2018.

19 Shoemark A, Boon M, Brochhausen C, et al. International consensus guideline for reporting transmission electron microscopy results in the diagnosis of primary ciliary dyskinesia (BEAT PCD TEM Criteria). Eur Respir J 2020; 55: 1900725.

20 Piatti G, De Santi MM, Farolfi A, et al. Exacerbations and Pseudomonas aeruginosa colonization are associated with altered lung structure and function in primary ciliary dyskinesia. BMC Pediatr 2020; 20: 158.

21 Alanin MC, Aanaes K, Høiby N, et al. Sinus surgery can improve quality of life, lung infections, and lung function in patients with primary ciliary dyskinesia. Int Forum Allergy Rhinol 2017; 7: 240-247.

22 Sommer LM, Alanin MC, Marvig RL, et al. Bacterial evolution in PCD and CF patients follows the same mutational steps. Sci Rep 2016; 6: 28732.

23 Davis SD, Ferkol TW, Rosenfeld M, et al. Clinical features of childhood primary ciliary dyskinesia by genotype and ultrastructural phenotype. Am J Respir Crit Care Med 2015; 191: 316-324.

24 Noone PG, Leigh MW, Sannuti A, et al. Primary ciliary dyskinesia: diagnostic and phenotypic features. Am J Respir Crit Care Med 2004; 169: 459-467.

25 Maglione M, Bush A, Nielsen KG, et al. Multicenter analysis of body mass index, lung function, and sputum microbiology in primary ciliary dyskinesia. Pediatr Pulmonol 2014; 49: 1243-1250.

26 Frija-Masson J, Bassinet L, Honoré I, et al. Clinical characteristics, functional respiratory decline and follow-up in adult patients with primary ciliary dyskinesia. Thorax 2017; 72: 154-160.

27 Daley $\mathrm{CL}$, laccarino JM, Lange $\mathrm{C}$, et al. Treatment of nontuberculous mycobacterial pulmonary disease: an official ATS/ERRS/ESCMID/IDSA Clinical Practice Guideline. Eur Respir J 2020; 56: 2000535.

28 Muhlebach MS, Beckett V, Popowitch E, et al. Microbiological efficacy of early MRSA treatment in cystic fibrosis in a randomised controlled trial. Thorax 2017; 72: 318-326.

29 Hansen CR. Stenotrophomonas maltophilia: to be or not to be a cystic fibrosis pathogen. Curr Opin Pulm Med 2012; 18: 628-631.

30 Dalbøge CS, Hansen CR, Pressler T, et al. Chronic pulmonary infection with Stenotrophomonas maltophilia and lung function in patients with cystic fibrosis. J Cyst Fibros 2011; 10: 318-325.

31 Amin R, Jahnke N, Waters V. Antibiotic treatment for Stenotrophomonas maltophilia in people with cystic fibrosis. Cochrane Database Syst Rev 2020; 3: CD009249.

32 Pedersen $\mathrm{H}$, Mygind N. Absence of axonemal arms in nasal mucosa cilia in Kartagener's syndrome. Nature 1976; 262: 494-495.

33 Afzelius BA. A human syndrome caused by immotile cilia. Science 1976; 193: 317-319.

34 Kobbernagel HE, Buchvald FF, Haarman EG, et al. Efficacy and safety of azithromycin maintenance therapy in primary ciliary dyskinesia (Bestcilia): a multicentre, double-blind, randomised, placebo-controlled phase 3 trial. Lancet Respir Med 2020; 8: 493-505.

35 Paff T, Daniels JM, Weersink EJ, et al. A randomised controlled trial on the effect of inhaled hypertonic saline on quality of life in primary ciliary dyskinesia. Eur Respir J 2017; 49: 1601770.

36 Boucher RC. New concepts of the pathogenesis of cystic fibrosis lung disease. Eur Respir J 2004; 23: 146-158.

37 Floto RA, Olivier KN, Saiman L, et al. US Cystic Fibrosis Foundation and European Cystic Fibrosis Society Consensus Recommendations for the Management of Non-Tuberculous Mycobacteria in Individuals with Cystic Fibrosis: executive summary. Thorax 2016; 71: 88-90.

38 Recio R, Brañas P, Martínez MT, et al. Effect of respiratory Achromobacter spp. infection on pulmonary function in patients with cystic fibrosis. J Med Microbiol 2018; 67: 952-956.

39 Tetart M, Wallet F, Kyheng M, et al. Impact of Achromobacter xylosoxidans isolation on the respiratory function of adult patients with cystic fibrosis. ERJ Open Res 2019; 5: 00051-2019.

40 Cools P, Ho E, Vranckx K, et al. Epidemic Achromobacter xylosoxidans strain among Belgian cystic fibrosis patients and review of literature. BMC Microbiol 2016; 16: 122.

41 Gabrielaite M, Bartell JA, Nørskov-Lauritsen N, et al. Transmission and antibiotic resistance of Achromobacter in cystic fibrosis. J Clin Microbiol 2021; 59: e02911-20.

42 Hoo ZH, Hitchcock L, Curley R, et al. A comparison of the CFHH criteria against the Leeds criteria in determining the Pseudomonas aeruginosa status among adults with cystic fibrosis. Respir Med 2020; 171 : 106103.

43 Kalferstova L, Vilimovska Dedeckova K, Antuskova M, et al. How and why to monitor Pseudomonas aeruginosa infections in the long term at a cystic fibrosis centre. $J$ Hosp Infect 2016; 92: 54-60.

44 Pedersen SS, Koch C, Høiby N, et al. An epidemic spread of multiresistant Pseudomonas aeruginosa in a cystic fibrosis centre. J Antimicrob Chemother 1986; 17: 505-516.

45 Marvig RL, Dolce D, Sommer LM, et al. Within-host microevolution of Pseudomonas aeruginosa in Italian cystic fibrosis patients. BMC Microbiol 2015; 15: 218.

46 Smyth AR, Bell SC, Bojcin S, et al. European Cystic Fibrosis Society Standards of Care: Best Practice Guidelines. J Cyst Fibros 2014; 13: Suppl 1, S23-S42. 
47 Hill AT, Sullivan AL, Chalmers JD, et al. British Thoracic Society Guideline for Bronchiectasis in Adults. BMJ Open Respir Res 2018; 5: e000348.

48 Pittet D, Allegranzi B, Boyce J, et al. The World Health Organization Guidelines on Hand Hygiene in Health Care and their consensus recommendations. Infect Control Hosp Epidemiol 2009; 30: 611-622.

49 Wood ME, Stockwell RE, Johnson GR, et al. Face masks and cough etiquette reduce the cough aerosol concentration of Pseudomonas aeruginosa in people with cystic fibrosis. Am J Respir Crit Care Med 2018; 197: 348-355.

50 Stockwell RE, Wood ME, He C, et al. Face masks reduce the release of Pseudomonas aeruginosa cough aerosols when worn for clinically relevant periods. Am J Respir Crit Care Med 2018; 198: 1339-1342.

51 Rowbotham NJ, Palser SC, Smith SJ, et al. Infection prevention and control in cystic fibrosis: A systematic review of interventions. Expert Rev Respir Med 2019; 13: 425-434.

52 Bundgaard $\mathrm{H}$, Bundgaard JS, Raaschou-Pedersen DET, et al. Effectiveness of adding a mask recommendation to other public health measures to prevent SARS-CoV-2 infection in Danish mask wearers: a randomized controlled trial. Ann Intern Med 2020; 174: 335-343. 